$\begin{array}{cl}\begin{array}{c}\text { Revue } \\ \text { de } / \text { histoire } \\ \text { des religions }\end{array} & \text { Revue de l'histoire des religions } \\ & \begin{array}{l}1 \mid 2007 \\ \text { Varia }\end{array}\end{array}$

\title{
Inquisition et judaïsants dans l'Amérique espagnole (1569-1649)
}

Carthagène des Indes au temps des réseaux

\section{Ricardo Escobar Quevedo}

\section{CpenEdition}

\section{Journals}

Édition électronique

URL : http://journals.openedition.org/rhr/5248

DOI : $10.4000 /$ rhr.5248

ISSN : 2105-2573

\section{Éditeur}

Armand Colin

Édition imprimée

Date de publication : 1 janvier 2007

Pagination : 47-59

ISBN : 978-2200-92332-7

ISSN : 0035-1423

\section{Référence électronique}

Ricardo Escobar Quevedo, «Inquisition et judaïsants dans l'Amérique espagnole (1569-1649) », Revue

de l'histoire des religions [En ligne], 1 | 2007, mis en ligne le 01 mars 2010, consulté le 19 avril 2019

URL : http://journals.openedition.org/rhr/5248; DOI : 10.4000/rhr.5248 


\section{POSITIONS DE THÈSES}

\section{Inquisition et judaïsants dans l'Amérique espagnole (1569-1649) Carthagène des Indes au temps des réseaux*}

Notre étude vise à présenter la première typologie multidimensionnelle du marranisme ibéro-américain (dans la mesure où elle prend en compte, outre la racine religieuse de ce phénomène, ses dimensions culturelles, économiques et sociales), ainsi que les modalités d'association des groupes implantés en Amérique espagnole et l'articulation de leurs réseaux familiaux, religieux et commerciaux avec la métropole, l'Afrique et les juiveries européennes.

Le cadre spatio-temporel retenu couvre les territoires placés sous la juridiction des trois tribunaux du Saint-Of ce établis en Amérique espagnole (Lima, Mexico et Carthagène des Indes), pendant la période 1569-1649. La première date correspond à la fondation du tribunal de Lima et donc aux débuts du Saint-Of ce de l'Inquisition au Nouveau Monde, bien que la répression à l'encontre des groupements marranes ne soit perceptible qu'après 1590. La seconde date (1649) a aussi sa part d'arbitraire : l'autodafé célébré au Mexique

* Thèse de Doctorat d'Histoire, sous la direction de M. Charles Amiel, présentée à l'École des Hautes Études en Sciences Sociales (EHESS) le 9 novembre 2005 (2 vol., 498 p.) - Membres du Jury : MM. les Professeurs Charles Amiel (EHESS), Jonathan Israel (Institute for Advanced Studies, Princeton), Bernard Lavallé (Université de Paris III - Sorbonne Nouvelle), Rodolfo de Roux (Université de Toulouse - Le Mirail). - (Mention Très honorable, avec les félicitations du jury à l'unanimité.) 
en avril 1649 marque la $\mathrm{n}$ de l'of fensive inquisitoriale contre ce que l'on a appelé les "Grandes Complicités » américaines, mais nous avons avancé dans le siècle a n d'observ er le ralentissement progressif de l'activité inquisitoriale.

Nous sommes bien conscient cependant que les circonscriptions et les époques sont uctuantes et imprécises, que les interférences et les transferts sont multiples et complexes et qu'enserrer le marranisme dans des frontières nationales ou des districts inquisitoriaux risque de simpli er un phénomène qui se décline à l'échelle planétaire. Car les bouleversements produits par l'expulsion d'Espagne, en 1492, avaient accouché d'une nouvelle donne. L'émancipation juridique des nouveaux-chrétiens et l'installation de groupes juifs dans la Méditerranée orientale permettent à ces nouveaux acteurs d'intervenir dans les trois axes des routes commerciales (l'Espagne, l'Empire ottoman et le Portugal), autour desquels, à l'aube du XVI ${ }^{\mathrm{e}}$ siècle, se réorganise le mercantilisme. Désormais, juifs et nouveaux-chrétiens - issus du monde ibérique et souvent parents ou alliés - se trouvent en position de participer au commerce de longue distance.

Pionniers d'un commerce qui brasse des marchandises venues des quatre coins du globe, ces hommes sont des voyageurs infatigables : marchands un jour dans les faubourgs de Manille, on les trouve quelques mois plus tard à Cuzco, à Lisbonne ou à Acapulco ; tel autre, tra quant d'escla ves entre Carthagène des Indes et l'embouchure du Niger, va être condamné par le tribunal de Lima, avant de devenir un négociant prospère à Rouen. Cependant, la plupart de ces marchands rompus au commerce transocéanique vont nir par perdre, en raison de leur vie nomade, le lien social avec les groupes judaïsants ; leurs vies d'aventuriers n'offrent donc pas à l'historien des phénomènes religieux une assise suf samment solide.

Pour cette raison, nous avons privilégié l'observation des migrations familiales plus ou moins organisées qui vont permettre la sauvegarde du marranisme. Parmi les centaines de cas jugés en Amérique, il nous fallait dégager un modèle représentatif de la diaspora, cohérent par son origine et par une pratique stable du marranisme. Le dépouillement des 617 cas de judaïsants condamnés par l'ensemble du Saint-Of ce américain entre 1569 et 1669 nous a 
permis d'identi er un groupe témoin : il s'agit de celui issu du district de Castelo Branco, dans la Beira Baixa portugaise. Ce territoire qui borde la frontière avec l'Espagne abritait depuis le Moyen Âge un collectif d'hommes et de femmes liés par des mariages endogames et par la pratique du judaïsme. Avec l'interdiction de la religion mosaïque et la création du Saint-Of ce portug ais, une partie importante du groupe néo-chrétien de la contrée va continuer ses pratiques ancestrales, cette fois dans le secret.

Ces groupements crypto-juifs établis à Castelo Branco vont devenir le vivier principal du marranisme américain et illustrent bien la continuité des vagues migratoires provoquées par le durcissement de la politique inquisitoriale pendant le $\mathrm{XVI}^{\mathrm{e}}$ et le $\mathrm{XVII}{ }^{\mathrm{e}}$ siècles. L'offensive lancée dans les années 1580 amorce l'exode vers l'Espagne, puis, au cours de la décennie 1620, lorsque le tribunal de Lisbonne redouble la cadence répressive, la ruée des nouveaux-chrétiens est telle que les villes de la contrée vont se dépeupler.

Le dépouillement de la documentation inquisitoriale américaine concernant les dernières décennies $\mathrm{du} \mathrm{XVI}^{\mathrm{e}}$ siècle nous a permis de mettre au jour cette migration, capitale pour restituer l'histoire du marranisme implanté en Amérique. Acculées par la persécution inquisitoriale, plusieurs lignées néo-chrétiennes vont traverser la frontière pour s'établir à Séville. Cependant, l'exode vers le port andalou ne représente pas une déchirure. Bien au contraire, la vie urbaine facilite les contacts religieux et sociaux entre des judaïsants venus des quatre coins du royaume lusitanien : les pratiques collectives retrouvent leur vigueur, les mariages entre lignées judaïsantes se multiplient. La promulgation de l'Édit papal de pardon, en 1605, va offrir le répit nécessaire à l'enracinement du marranisme portugais à Séville, d'où il va rayonner sur toute la diaspora ibéro-américaine. Désormais, Séville va devenir la seconde matrice du marranisme américain et sa seconde mémoire.

La véritable dispersion ne viendra qu'au Nouveau Monde, continent aux espaces presque in nis, où la séparation géographique va produire inéluctablement l'éclatement des pratiques. Cependant, ces deux racines, la portugaise et la sévillane, vont rester ancrées dans la mémoire des judaïsants qui partiront construire une nouvelle 
vie outre-Atlantique. Pendant plus d'un demi-siècle, les inquisiteurs américains continueront à dépoussiérer des vieilles preuves d'hérésie : les initiations à la loi de Moïse dans les champs d'oliviers de Castelo Branco, les jeûnes dans les arrière-boutiques de la rue Sierpe, l'attente de cette étoile scintillant sur le Guadalquivir qui annonçait la n du Kippour.

$* * *$

Regardons de plus près les grands traits des pratiques religieuses. L'étude que nous avons menée sur le phénomène marrane américain con rme la nécessité d'intégrer plusieurs v ariables qui favorisent ou gênent son épanouissement. Tout d'abord, la reproduction au Nouveau Monde d'un modèle familial attaché à la transmission du legs religieux. Si l'origine portugaise rassemble la grande majorité des crypto-juifs américains, la constitution d'un groupe familial différencie les pratiques. Car, depuis l'interdiction du judaïsme, la famille était devenue le lieu privilégié de la transmission, la forteresse de la mémoire. En dépit de leur proximité géographique, les groupements implantés au Nouveau Monde évoluent de manière divergente. En Nouvelle-Espagne, le groupe mosaïque réussit à reproduire le modèle familial et l'exercice traditionnel du marranisme, générant une dynamique orientée vers la croissance démographique et la généralisation des pratiques religieuses.

C'est bien le clivage majeur qui sépare les pratiques des marranes en Amérique espagnole, car bien que quelques couples s'installent au Pérou et en Nouvelle-Grenade, seul le groupe mexicain réussit une véritable implantation familiale. La femme judaïsante, relais traditionnel du marranisme ibérique, est presque introuvable au sud de l'Amérique espagnole, tandis qu'elle est omniprésente au Mexique : elle s'y attache à la sauvegarde des pratiques collectives ancestrales - parfois contre la volonté des hommes - et garantit la survie générationnelle du marranisme. Tant que la cellule familiale réussit à se reproduire, le marranisme persévère. La continuité des alliances endogames au Mexique resserre le collectif et assure une assise propice à la transmission de l'héritage religieux. Dif cile, lorsqu'un enfant grandit au sein d'une lignée judaïsante comme celle originaire 
de Castelo Branco, de rompre avec ce qui devient la norme, car la force d'un ensemble homogène réussit à inverser les valeurs. Dans ce microcosme familial, le judaïsme est la norme, le christianisme l'anomalie.

Une deuxième variable, beaucoup plus complexe, est l'environnement social qui entoure les groupes marranes et le degré d'isolement qui en découle. Sous la forme d'une pratique clandestine du judaïsme, le marranisme reste un phénomène global. Cependant, on croit pouvoir déceler deux époques. Lorsque le marranisme de souche ibérique, coupé depuis un siècle de la littérature juive et du contact avec des autorités rabbiniques, commence à montrer des signes d'affaiblissement, les contacts avec le judaïsme européen revitalisent les pratiques. Si les premières familles arrivées au Mexique pratiquent encore, vers 1580, le judaïsme hérité de leurs aïeux, les connaissances véhiculées par des voyageurs venus des juiveries européennes vont donner au groupe un second souf e dans les années qui sui vent.

Car les pratiques des crypto-juifs installés en Amérique espagnole sont loin d'être gées. Bien qu'elles conserv ent une composante ibérique fondatrice, elles sont enrichies par des contacts, même s'ils sont sporadiques, avec les juiveries et les groupements marranes européens. On peut en effet constater que la matière religieuse des judaïsants américains est souvent le produit de plusieurs foyers marranes - ibériques et français - qui se complètent ou se juxtaposent ; on constate également l'arrivée au Nouveau Monde de voyageurs venus d'Italie ou de Hollande qui interviennent dans l'évolution des pratiques religieuses. De ce fait, on se trouve face à un corpus doctrinal relativement hétéroclite qui peut combiner la tradition marrane et le judaïsme normatif. Ce sont les inquisiteurs mexicains eux-mêmes qui constatent l'arrivée de juifs venus des "synagogues » européennes et l'accueil favorable que leur réservent les judaïsants fervents. L'in uence de marranes v enus de France est tout aussi importante et constante : Diego Pérez de Albuquerque et Jorge Jacinto Bazán, arrêtés au Mexique, grandirent en France, où ils avaient été circoncis. C'est à Bordeaux et à Saint-Jean-de-Luz que Leonor Núñez s'imprègne du judaïsme normatif pendant dix ans, avant de s'embarquer vers le Mexique. 
Ainsi, par sa con guration $\mathrm{f}$ amiliale, le marranisme mexicain est presque la copie conforme de l'ibérique. Mais qu'en est-il du sud de l'Amérique espagnole ? En fait, l'évolution n'est pas la même lorsqu'il s'agit d'un groupe structuré, comme le mexicain, que lorsqu'il s'agit des conglomérats constitués très largement d'hommes seuls comme ceux de la Nouvelle-Grenade et du Pérou. Si les voyageurs venus de juiveries européennes trouvent au Mexique une communauté organisée et avide de connaissances, ceux qui, beaucoup moins nombreux, arrivent dans les territoires espagnols situés au sud du continent, ne semblent pas avoir d'incidence sur l'évolution des pratiques religieuses : Baltasar Araújo Coronel, circoncis à Venise et élevé dans le judaïsme à Istanbul, ne cherche que l'anonymat et l'oubli lorsqu'il s'installe dans l'arrière-pays néo-grenadin.

On peut même déceler des « dérives » tragiques, comme cette étrange vocation au martyre, dont les ressorts restent dif ciles à cerner, montrée par une poignée de judaïsants arrêtés au sud de l'Amérique espagnole entre la $\mathrm{n}$ du $\mathrm{XVI}^{\mathrm{e}}$ siècle et le début du XVII ${ }^{\mathrm{e}}$. À cette époque, le contraste entre les groupes marranes américains est déjà très marqué. Tandis que ces hommes errent entre la côte paci que et les hauts-plateaux andins, les marranes me xicains pratiquent collectivement les rites crypto-juifs. Malgré le harcèlement du Saint-Of ce, la vie familiale s'installe alors dans une certaine normalité, les célébrations et les moments de bonheur sont perceptibles : hommes, femmes et enfants réunis s'adonnent aux rites et aux commémorations, tandis que la harpe de Manuel Lucena accompagne la lecture des psaumes. Même lorsque la mort d'un judaïsant plonge le groupe mexicain dans l'amertume, ils trouvent réconfort grâce à la consolation ouverte et collective. Certains parmi eux, comme Ruy Díaz Nieto, apportent de surcroît les connaissances des juiveries italiennes et l'espoir d'un judaïsme libre du joug inquisitorial.

$* * *$

Grâce à des réseaux commerciaux étendus à une échelle planétaire, les marchands néo- chrétiens et juifs vont contribuer à l'émergence d'une modernité économique en Occident, d'une mondialisation avant l'heure. Une modernité contrastée cependant dans le cas 
américain, où le progrès du système mercantile occulte des formes d'exploitation attachées au passé : la réinvention d'un esclavage à l'ancienne. Le commerce d'esclaves africains vers les Amériques, une des seules activités qui garantissent la promotion sociale des Portugais après l'union des Couronnes ibériques, devient la chasse gardée des nouveaux-chrétiens. L'asiento - un contrat accordé par la Couronne permettant d'introduire un certain nombre d'esclaves au Nouveau Monde - dont béné cient les Portugais entre 1595 et 1640 est une affaire maîtrisée de bout en bout de la chaîne par des nouveaux-chrétiens, et bien que la sécession portugaise les ait éloignés temporairement du marché hispano-américain, l'essor de la communauté juive d'Amsterdam et la prise de Curaçao vont leur permettre de prendre pied de nouveau dans la Caraïbe.

L'énorme pouvoir économique qu'acquièrent les négriers nouveaux-chrétiens pendant l'asiento portugais résulte de l'ef cacité d'un réseau commercial disséminé des deux côtés de l'Atlantique, dont la charnière est Carthagène des Indes. Dans cet espace périphérique, les traditions ne tardent à partir à la dérive. Les déplacements à l'intérieur du continent africain ou lors de longues traversées transocéaniques empêchent le contact non seulement avec les autorités rabbiniques, mais aussi, ce qui était fondamental pour la préservation du marranisme, avec des femmes judaïsantes. Univers interlope par essence, la traite est une affaire d'hommes. Alors, ces judaïsants qui ottent dans l'uni vers afro-américain lié à la traite vont vivre en concubinage avec des noires ou des mulâtresses, avec lesquelles ils procréent des enfants mulâtres : un schéma de rupture avec le modèle endogame caractéristique du judaïsme médiéval qui se répète des deux côtés de 1'Atlantique.

La participation des nouveaux-chrétiens à toutes les modalités commerciales de la traite négrière va générer des métissages et promouvoir des conversions au judaïsme en Afrique et dans l'aire caraïbe ; les quelques cas d'esclaves convertis au judaïsme répertoriés par le Saint-Of ce américain ne sont que la partie émer gée de l'iceberg. L'inquiétude des inquisiteurs de Carthagène face à de nombreux esclaves qui embrassaient avec « témérité » la foi de leurs maîtres, semble montrer que le prosélytisme des négriers était loin 
d'être un phénomène marginal ; un métissage que les lançados portugais avaient amorcé très tôt en Afrique occidentale, ces terres de traite qui sont souvent le miroir de l'univers caraïbe.

La profusion de contacts avec la population africaine va aussi laisser son empreinte sur les mœurs des groupements marranes issus de Castelo Branco ; une évolution d'autant plus intéressante qu'il s'agit là d'un groupe issu d'un milieu rural, loin du cosmopolitisme qui entoure les nouveaux-chrétiens de Lisbonne ou de Porto. Déjà à Séville, les premiers contacts avec la population africaine, même s'ils sont à cette époque encore limités, vont bouleverser les traditions et générer des relations inédites. Loin de la province lusitanienne qui avait facilité la constitution d'un ensemble exclusivement néo-chrétien, le marranisme s'adapte, s'ouvre timidement à l'autre. Désormais, sous certaines conditions, l'exogène est admis ; la transmission par le sang, pilier de la politique d'exclusion menée par la Monarchie, imprègne l'imaginaire marrane, mais cette fois le mythe du sang génère des ouvertures. Ainsi, les enfants nés des relations entre un juif et une esclave sont susceptibles d'être convertis au judaïsme, car, comme ne cesse de le répéter la « dogmatiste » Blanca Enríquez, «tienen sangre nuestra ». Une lignée issue du métissage, celle d'Isabel, esclave guinéenne, et d'un nouveau-chrétien va devenir une de plus reconnues en Amérique : la lle, Esperanza Rodríguez, une mulâtresse, va adopter dès sa plus tendre enfance la religion juive, tout comme les petites- lles, Juana, née à Carthagène des Indes, et María, née à Acapulco, toutes les trois condamnées par le Saint-Of ce.

Dresser un pro 1 du phénomène marrane en Amérique semble impossible, tellement les pratiques varient d'un territoire à l'autre, d'une conjoncture à l'autre. Cependant, la documentation inquisitoriale nous permet d'esquisser quelques traits généraux des groupes marranes implantés au Nouveau Monde. Tout d'abord, par leur structure, ils ne forment pas un ensemble. Le modèle familial mexicain permet l'apparition d'une génération de marranes née sur le sol américain : parmi les judaïsants jugés en Amérique espagnole pendant la première moitié du XVII ${ }^{\mathrm{e}}$ siècle, 44 sont nés en Nouvelle-Espagne ; 
9 seulement voient le jour au Pérou (dont la moitié grandit en réalité au Mexique); à peine 2 naissent en Nouvelle-Grenade.

Un des moments forts de l'histoire du marranisme américain, la vague répressive de 1635-1649, nous permet de cerner un peu mieux la géographie des groupes marranes. L'absence de dénonciations contre des judaïsants mexicains, parmi les centaines extorquées lors de la « Grande Complicité » péruvienne (1635-1639), démontre que la séparation entre les groupes mexicain et péruvien est assez nette. Par contre, les rapports entre Lima et Carthagène sont très étroits, au point qu'on peut parler d'un seul et unique groupe, uni par les liens du commerce et de la parenté : dix-neuf témoignages extorqués à Lima contre des judaïsants de Carthagène vont arriver au tribunal néogrenadin, et un nombre similaire fera le chemin de retour. Dans les faits, la répression mexicaine aura plus de conséquences pour les judaïsants de Séville, tellement les liens commerciaux et familiaux entre les communautés andalouse et mexicaine sont forts et constants. Les groupes de Lima et de Carthagène se projettent aussi naturellement vers Séville, mais ils ont également des rapports étroits avec les enclaves portugaises d'Afrique : un nombre important des accusés interpellés au sud du continent débarque d'Angola, de Guinée ou de São Tomé, où, souvent, ils s'étaient livrés à des pratiques juives.

Le volume des procès instruits par le Saint-Of ce américain nous donne d'emblée une idée de la hiérarchie des tribunaux : entre 1570 et 1700 le tribunal de Mexico instruisit 1933 procès, le tribunal de Lima 1 176, et celui de Carthagène des Indes (pour la période 1610-1700) 731. Nos statistiques, en ce qui concerne les cas instruits à l'encontre de judaïsants, con rment cette proportion : le tribunal de Mexico prononça 379 sentences, celui de Lima 223, et celui de Carthagène 81.

La répression contre les judaïsants ne représente qu'un pourcentage modeste de l'activité globale de l'Of ce américain : $18 \%$ à Lima, $12 \%$ à Carthagène, près de $10 \%$ à Mexico. Cependant, ces chiffres sont trompeurs, car l'action du Saint-Of ce cible a vant tout le groupe néo-chrétien, et c'est sur lui que s'abat toute la force du châtiment. Les condamnations à mort ne représentent que $1,7 \%$ des sentences prononcées par le tribunal mexicain ; un pourcentage 
in me, certes, qui recoupe ceux d'autres trib unaux métropolitains. Mais les chiffres bruts n'ont pas de sens s'ils ne sont pas corrélés avec la typologie des délits, comme l'a bien établi Charles Amiel dans ses travaux, sur Goa en particulier. Voyons, par exemple, quelles sont les proportions par délit parmi les accusés qui subirent la peine capitale : sur 40 sentences au bûcher prononcées par le tribunal mexicain entre 1569 et 1669 contre des personnes vivantes, les judaïsants représentent $75 \% ; 12,5 \%$ sont des protestants, et les $12,5 \%$ restants correspondent à des hérétiques non précisés et à un faux prêtre. Pour ce qui est des relaxés en ef gie (103 indi vidus), $99 \%$ sont des judaïsants. L'état de la recherche est encore trop peu avancé, particulièrement en ce qui concerne une étude systématique des sentences, pour y voir clair ; cependant, les judaïsants semblent aussi largement majoritaires parmi les condamnés aux galères, à la prison perpétuelle et au bannissement.

Les sentences du tribunal de Lima con rment les tendances du Mexique. Entre la fondation du tribunal, en 1569, et l'année 1664, 31 condamnations au bûcher furent prononcées contre des personnes vivantes : $71 \%$ étaient des judaïsants, $19 \%$ des protestants, $6 \%$ des accusés de « propositions », et $3 \%$ étaient accusés d'appartenir à la secte des «alumbrados». La totalité des condamnés à mort par contumace étaient des judaïsants ; une sentence qui était loin d'être symbolique, car elle permettait au tribunal de mettre la main sur les biens du défunt ou du fugitif condamné, et marquait sa descendance du sceau de l'ignominie. Les conclusions que nous pouvons tirer du tribunal de Carthagène sont très limitées, car pendant le XVII ${ }^{\mathrm{e}}$ siècle il prononça seulement deux condamnations à mort contre des personnes vivantes : une contre un judaïsant et une contre un protestant ; les deux ef gies brûlées pendant cette période représentaient des judaïsants.

D'après nos statistiques, entre 1569 et 1669 le Saint-Of ce américain brûla en personne 54 personnes accusées de judaïsme (7,9\% du nombre de sentences prononcées à l'encontre des judaïsants), tandis que 116 le furent en ef gie $(16,9 \%)$. Force est de constater que la majorité des accusés furent réconciliés, absous ou graciés. Si toute la dureté du châtiment s'appliquait aux relaps, le Saint-Of ce réintégrait dans le giron de l'Église ceux qui montraient des signes 
de repentance. Souvent, des anciens réconciliés retrouvèrent leur prestige une fois leurs peines accomplies et devinrent des hommes de con ance de la Cour espagnole.

Des bilans néanmoins à manier avec précaution. Bien que le Saint-Of ce soit une institution monolithique et $\mathrm{v}$ erticale régie par un cadre juridique, l'éloignement des tribunaux américains octroie une certaine autonomie aux inquisiteurs. Plus encore, il faut tenir compte de l'environnement qui entoure chacun des tribunaux. Ce n'est pas le même Saint-Of ce qui agit à Lima ou à Me xico, deux pôles de la colonisation américaine dotés d'universités et capitales de vice-royautés, que celui implanté à Carthagène des Indes : immergés dans un monde de contrebandiers, où 1200 européens cohabitaient avec 12000 esclaves africains, les agents du tribunal néo-grenadin vont souvent succomber aux pires dérives.

Toute l'originalité du tribunal de Carthagène des Indes apparaît au grand jour dans les rapports rédigés par l'inspecteur Pedro Medina Rico au milieu du $\mathrm{XVII}^{\mathrm{e}}$ siècle. Une institution gangrenée par la corruption, au milieu d'une ville qui semblait égarée dans le temps. C'est bien Pedro Medina Rico lui-même qui concluait une de ses lettres avec une dernière plainte, anodine mais si parlante de l'état pitoyable de cette terre qui avait ni par a voir raison des desseins de la Monarchie : à Carthagène - écrivait-il -, il n'y a même pas une horloge, uniquement des soldats de garnison qui, une cloche à la main, se chargent de sonner l'heure lorsque bon leur semble. Alors, rares sont les moments où les hommes de l'Of ce se trouv ent au complet : ministres et of ciers rechignent à g agner le tribunal à l'heure, les uns par paresse, les autres pour ne pas avoir à attendre leurs collègues, impatients tous, lorsqu'ils sont réunis, d'entendre que l'arbitraire cloche sonne la $\mathrm{n}$ de la journée.

\section{$* * *$}

Notre étude aboutit ainsi à diverses conclusions, dont nous résumons les plus importantes:

- Il y a une nette différence de style entre les trois ensembles territoriaux américains placés sous la surveillance du Saint-Of ce, tant pour la pratique religieuse que pour les formes d'articulation des groupements néo-chrétiens. Au modèle familial développé 
au Mexique, garant de la reproduction générationnelle du groupe et de la promotion des pratiques collectives, s'oppose celui des groupements de la Caraïbe et du sud de l'Amérique espagnole constitués presque exclusivement d'hommes.

- Grâce à une étude généalogique minutieuse, nous avons prouvé la prédominance portugaise dans l'origine géographique des cryptojuifs américains, mais aussi l'importance du district de Castelo Branco, berceau de quelques-unes des principales lignées marranes.

- Ces familles, issues d'un milieu rural, vont entamer dès la $\mathrm{n}$ du $\mathrm{XVI}^{\mathrm{e}}$ siècle un mouvement migratoire ponctué par les offensives du Saint-Of ce portug ais. En quelques années, Séville se consolide comme l'un des bastions du marranisme ibérique et sa porte de sortie vers le Nouveau Monde.

- Le recoupement des fonds inquisitoriaux et administratifs nous permet de constater que, pendant l'asiento octroyé par la Couronne espagnole aux Portugais (1595-1640), les nouveaux-chrétiens ont exercé un véritable monopole sur la traite des Noirs. Cette proximité va générer au passage un métissage original des marchands néo-chrétiens avec des femmes noires, qui marque une rupture avec le mariage endogamique typique du marranisme ibérique.

- Bien que les pratiques marranes conservent une composante ibérique fondatrice, la matière religieuse est enrichie par les contacts avec les juiveries européennes (Pays-Bas, Italie et groupements marranes tolérés de France : Rouen et Sud-Ouest). De ce fait, on se trouve face à un corpus doctrinal qui peut combiner la tradition marrane et le judaïsme normatif.

- Si le mouvement inquisitorial américain présente une courbe commune aux trois tribunaux (deux fortes répressions séparées par un répit dû au Bref papal de 1605), l'application de la politique inquisitoriale montre des variations importantes (comme signalé plus haut) sur l'ensemble du territoire.

$* * * *$

Partis des contrées perdues au $\mathrm{n}$ fond de la pro vince portugaise, les groupements marranes vont être confrontés à un monde nouveau. 
Un siècle après l'interdiction du judaïsme, ils restent néanmoins attachés à cette religion ancestrale que le temps et la persécution risquent chaque jour de condamner à l'oubli : dans ces terres d'outre-mer, le peuple du Livre est devenu celui de la mémoire. Le groupe de Castelo Branco n'est qu'un échantillon de l'univers marrane, mais il illustre bien la diversité des destins que forge l'Amérique : au sein d'une même lignée se côtoient le martyr et le renégat, le puissant et la couturière, le négrier et le médecin. Aux extrémités de cet éventail se trouvent deux personnages emblématiques du tiraillement entre les deux religions, de l'affrontement entre l'assimilation et la persévérance : le mouchard et la « dogmatiste».

L'Amérique coloniale n'est pas avare de paradoxes : parfois le persécuté vit de la misère des esclaves, des judaïsantes passionnées multiplient les amours libertines, évêques et inquisiteurs se font soigner par la magie de sorcières reconnues. Continent exubérant où les frontières bâties dans la lointaine métropole deviennent oues. Dans ce Nouveau Monde en construction, le destin des judaïsants et des inquisiteurs est toujours imbriqué : des antagonistes liés par une dialectique qui ne cesse de les opposer mais qui parfois les attire. Des inquisiteurs redoutables deviendront à Carthagène des Indes des amis dévoués et des partenaires sûrs des anciens condamnés ; au Mexique, des judaïsants fervents deviendront avec le temps des collaborateurs zélés du Saint-Of ce, des crypto-chrétiens parmi les crypto-juifs. Le jeu de miroirs est troublant : de même que des lignées judaïsantes pérennisent la tradition juive, l'Inquisition dispose aussi de familles vouées à la lutte contre l'hérésie, comme celle des Mañozca, qui donna dix-huit de ses enfants aux troupes de l'Of ce. En n, comble de l'ironie, la mémoire du marranisme v a être sauvée par les fonds du Saint-Of ce, institution créée avec la nalité exclusive de la supprimer* ${ }^{* *}$.

** La thèse a été acceptée pour publication par les Presses de l'Université Paris-Sorbonne (PUPS). L'auteur est actuellement Chargé de conférences à l'École pratique des Hautes Études (Sciences religieuses), Paris. [NdlR] 
